\title{
Hepatic Steatosis
}

National Cancer Institute

\section{Source}

National Cancer Institute. Hepatic Steatosis. NCI Thesaurus. Code C150596.

A morphologic finding indicating intracytoplasmic fat accumulation in the liver parenchyma. 